\title{
MAGNETO-ROTATORY COMPRESSIBLE COUPLE-STRESS FLUID HEATED FROM BELOW IN POROUS MEDIUM
}

\author{
CHANDER BHAN MEHTA
}

Department of Mathematics, Centre of Excellence, Sanjauli, Shimla, India, e-mail: chanderbmehta@gmail.com

\begin{abstract}
The study is aimed at analysing thermal convection in a compressible couple stress fluid in a porous medium in the presence of rotation and magnetic field. After linearizing the relevant equations, the perturbation equations are analysed in terms of normal modes. A dispersion relation governing the effects of rotation, magnetic field, couple stress parameter and medium permeability have been examined. For a stationary convection, the rotation postpones the onset of convection in a couple stress fluid heated from below in a porous medium in the presence of a magnetic field. Whereas, the magnetic field and couple stress postpones and hastens the onset of convection in the presence of rotation and the medium permeability hastens and postpones the onset of convection with conditions on Taylor number. Further the oscillatory modes are introduced due to the presence of rotation and the magnetic field which were non-existent in their absence, and hence the principle of exchange stands valid. The sufficient conditions for nonexistence of over stability are also obtained.
\end{abstract}

Key words: compressible couple-stress fluid, thermal convection, porous medium, rotation, magnetic field

\section{INTRODUCTION}

The theory of couple-stress fluid has been formulated by Stokes [17]. The theory, according to Stokes allows for polar effects such as the presence of couple-stresses and body couples. The theory has been applied to the study of some lubrication problems. According to the theory, couple-stresses are found to appear in noticeable magnitudes in fluids with very large molecules. Since the long chain hyaluronic acid molecules are found as additives in synovial fluid, Walicki and Walicka [19] modelled synovial fluid as a couple-stress fluid in human joints. One of the applications of couple-stress fluid is, therefore, its use in the study of the mechanisms of lubrication of synovial joints, which has become the object of scientific research. A human joint is a dynamically loaded bearing, which has articular cartilage functioning as the bearing and synovial fluid as the lubricant. When a fluid film is generated, squeeze-film action provides a considerable protection to the cartilage surface. The shoulder, hip, knee and ankle joints are loaded with synovial fluids of the human body and these joints have a low friction coefficient and negligible wear.

A good account of the effects of rotation on the layer of Newtonian fluid heated from below has been given by Chandrasekhar [3]. Brakke [2] explained a double-diffusive instability that occurs when a solution of a slowly diffusing protein is layered over a denser solution of a more rapidly diffusing sucrose. Nason et al. [7] found that this instability, which is deleterious to certain biochemical separations, can be suppressed by rotation in the ultracentrifuge. For the thermal convection problem, the Boussinesq approximation has been used, which is well justified in the case of incompressible fluids. The thermal instability of a fluid layer with maintained adverse temperature gradient when heated from below, plays an important role in geophysics, interior of the Earth, oceanography and atmospheric physics, etc. The use of Boussinesq approximation has been made throughout, which states that the density may be treated as constant in all the terms in the equation of motion except the external force term. Sharma [10] has considered the effect of rotation and magnetic field on the thermal instability in compressible fluids. The fluid has been considered to be Newtonian in all the above studies. Sharma and Sharma [12] have studied the couple-stress fluid heated from below in porous mediums. An electrically conducting couple-stress fluid heated from below in a porous medium in the presence of a magnetic field and rotations have been studied by Sunil et al. [18]. The use of magnetic field is being made for the clinical purposes in detection and cure of certain diseases with the help of magnetic field devices/instruments. 
Sharma and Thakur [13] have studied the thermal convection in couple-stress fluid in porous medium in hydromagnetics. Sharma and Mehta [11] studied the thermosolutal convection in compressible rotating couple stress fluid and found that the couple stress viscosity postponed as well as hastened the onset of convection depending on rotation parameter. Rudraiah and Chandrashekara [9] studied the effect of couple stress on the growth rate of Rayliegh-Taylor instability at the interface in the finite couple stress fluid in which they found that the effect of couple stress parameter reduces the growth rate considerably when compared to their classical growth rate in the absence of the couple stress. Kumar [5] found that in the absence of rotation, medium permeability has the destabilized effect, whereas magnetic field and couple stress parameter have stabilizing effect. Banyal [1] studied a mathematical theorem on set of the stationary convection in the couple stress fluid. Singh and Mehta [15] studied the effect of uniform vertical magnetic field and rotation in a layer, electrically conducting stress fluid in a porous medium and observed that the rotation had postponed the onset of convection, whereas medium permeability and couple-stress parameter had postponed as well as hastened the onset of convection. Rana [8] studied the onset of thermal convection in couple-stress fluid in hydromagnetics saturating a porous medium and established the stabilizing character of couple-stress parameter.

The study is motivated by a model of synovial fluid. The synovial fluid is a natural lubricant of joints of the vertebrates. The detailed description of the joint lubrication has very important practical implications. Practically all diseases of joints are caused by or connected with a malfunction of the lubrication. The external efficiency of the physiological joint lubrication is caused by more mechanisms. The synovial is due to the content of the hyaluronic acid, a fluid of high viscosity (a gel). A layer of such fluid heated from below in the presence of magnetic field may find applications in physiological processes such as in magnetic therapy and physiotherapy.

When the fluids are compressible, the equations governing the system become quite complicated. Spiegel and Veronis [16] have simplified the set of equations governing the flow of compressible fluids under the following assumptions:

(i) that the depth of the fluid layer is much smaller than the scale height as defined by them, if only motions of infinitesimal amplitude are considered and

(ii) the fluctuations in density, temperature and pressure introduced by the motion, do not exceed their static variations, in non-linear investigations.
Under the above assumptions, it was found that the equations governing convection in a compressible fluid are the same as those for incompressible fluids except that the static temperature gradient is replaced by its excess over the adiabatic one and $c_{v}$ is replaced by $c_{p}$.

With growing importance of non-Newtonian fluids, the present paper attempts to study the compressible, magneto-rotating, couple-stress fluid heated from below. The motivation for the present study are the decade old contradictions between theory and experiment (columnar instability) and the fact that the knowledge concerning fluid-particle mixture is not commensurate with their scientific and industrial importance. Further, motivation for this study is its usefulness in chemical technology, industry and biomechanics (e.g., physiotherapy) where compressibility of a fluid is an important aspect.

\section{FORMULATION OF THE PROBLEM AND PERTURBATION EQUATION}

Here, we consider an infinite, horizontal, incompressible, electrically conducting couple-stress fluid layer of thickness $d$, heated from below so, that the temperatures and densities at the bottom surface $z=0$ are $T_{0}$ and $\rho_{0}$ and at the upper surface $z=d$ are $T_{d}$ and $\rho_{d}$, respectively, and that a uniform temperature gradient $\beta\left(=\left|\frac{d T}{d z}\right|\right)$ is maintained. The gravity field $\vec{g}=(0,0,-g)$, a uniform vertical magnetic field $\vec{H}=$ $(0,0, H)$ and a uniform vertical rotation $\vec{\Omega}=(0,0, \Omega)$ act on the system. This fluid layer is flowing through an isotropic and homogenous porous medium of porosity $\varepsilon$ and medium permeability $k_{1}$.

Let $\rho, p, T, \eta, \mu_{e}$ and $\vec{q}=(u, v, w)$ denote the fluid density, pressure, temperature, resistivity, magnetic permeability and filter velocitym respectively. Then the momentum balance, mass balance and energy balance equations of couple-stress fluid through porous medium (Stokes [17], Joseph [4], Chandrasekhar [3]) are given by

$$
\begin{gathered}
\frac{1}{\varepsilon}\left[\frac{\partial \vec{q}}{\partial t}+\frac{1}{\varepsilon}(\vec{q} \cdot \nabla) \vec{q}\right]=-\nabla\left(\frac{p}{\rho_{0}}-\frac{1}{2}|\vec{\Omega} \times \vec{r}|^{2}\right) \\
+\vec{g}\left(1+\frac{\delta \rho}{\rho_{0}}\right)-\frac{1}{k_{1}}\left(v-\frac{\mu^{\prime}}{\rho_{0}} \nabla^{2}\right) \vec{q} \\
+\frac{\mu_{e}}{4 \pi \rho_{0}}(\nabla \times \vec{H}) \times \vec{H} \frac{2}{\varepsilon}(\vec{q} \times \vec{\Omega}),
\end{gathered}
$$




$$
\begin{gathered}
\nabla \cdot \vec{q}=0 \\
E \frac{\partial T}{\partial t}+(\vec{q} \cdot \nabla) T=\left(\beta-\frac{g}{c_{p}}\right) w+k \nabla^{2} T \\
\nabla \cdot \vec{H}=0 \\
\varepsilon \frac{d \vec{H}}{d t}=(\vec{H} \cdot \nabla) \vec{q}+\varepsilon \eta \nabla^{2} \vec{H}
\end{gathered}
$$

where $\frac{d}{d t}=\frac{\partial}{\partial t}+\varepsilon^{-1} \vec{q} \cdot \nabla$ stands for the convective derivative.

The equation of state is

$$
\rho=\rho_{0}\left[1-\alpha\left(T-T_{0}\right)\right]
$$

where the suffix zero refers to values at the reference level $z=0$. In writing equation (1), use has been made of Boussinesq approximation, which states that the density variations are ignored in all terms in the equation of motion except the external force term. The kinematic viscosity $v$, couple-stress viscosity $\mu^{\prime}$, magnetic permeability $\mu_{e}$, thermal diffusivity $\kappa$, electrical resistivity $\eta$, and coefficient of thermal expansion $\alpha$ are all assumed to be constants. Here, $E=\varepsilon+$ $(1-\varepsilon)\left(\frac{\rho_{s} c_{s}}{\rho_{0} c_{v}}\right)$ is a constant, while $\rho_{s}, c_{s}$ and $\rho_{0}, c_{v}$ stand for density and heat capacity of the solid (porous matrix) material and the fluid, respectively, and $\vec{r}=(x, y, z)$.

The basic motionless solution is

$$
\vec{q}=(0,0,0), \quad T=-\beta z+T_{0}, \quad \rho=\rho_{0}(1+\alpha \beta z) .
$$

Here, we use the linearized stability theory and the normal mode analysis method. Assume small perturbations around the basic solution, and let $\delta \rho, \delta p, \theta$, $\vec{q}=(u, v, w)$ and $\vec{h}=\left(h_{x}, h_{y}, h_{z}\right)$ denote respectively the perturbation in fluid density $\rho$, pressure $p$, temperature $T$, velocity $\vec{q}=(0,0,0)$ and the magnetic field $\vec{H}=(0,0, H)$. The change in density $\delta \rho$, caused mainly by the perturbation $\theta$ in temperature, is given by

$$
\delta \rho=-\alpha \rho_{0} \theta \text {. }
$$

Then the linearized perturbation equations of the couple-stress fluid reduce to

$$
\begin{aligned}
\frac{1}{\varepsilon} \frac{\partial \vec{q}}{\partial t}= & -\frac{1}{\rho_{0}}(\nabla \partial p)-\vec{g} \alpha \theta-\frac{1}{k_{1}}\left(v-\frac{\mu^{\prime}}{\rho_{0}} \nabla^{2}\right) \vec{q} \\
& +\frac{\mu_{e}}{4 \pi \rho_{0}}(\nabla \times \vec{h}) \times \vec{H}+\frac{2}{\varepsilon}(\vec{q} \times \vec{\Omega}),
\end{aligned}
$$

$$
\begin{gathered}
\nabla \cdot \vec{q}=0, \\
E \frac{\partial \theta}{\partial t}=\left(\beta-\frac{g}{c_{p}}\right) w+k \nabla^{2} \theta, \\
\nabla \cdot \vec{h}=0, \\
\varepsilon \frac{d \vec{h}}{d t}=(\vec{H} \cdot \nabla) \vec{q}+\varepsilon \eta \nabla^{2} \vec{h} .
\end{gathered}
$$

\section{THE DISPERSION RELATION}

Analyzing the disturbances into two-dimensional waves and considering disturbances characterized by a particular wave number, we assume that the perturbation quantities are of the form

$$
\begin{gathered}
{\left[w, h_{z}, \theta, \zeta, \xi\right]} \\
=[W(z), K(z), \Theta(z), Z(z), X(z)] \\
\times \exp \left(i k_{x} x+i k_{y} y+n t\right),
\end{gathered}
$$

where $k_{x}, k_{y}$ are wave numbers along $x$-and $y$-directions, respectively, $k\left(=\sqrt{k_{x}^{2}+k_{y}^{2}}\right)$ is the resultant wave number, and $n$ is the growth rate which is, in general, a complex constant. Let $\varsigma=\frac{\partial v}{\partial x}-\frac{\partial u}{\partial y}$ and $\xi=\frac{\partial h_{y}}{\partial x}-\frac{\partial h_{x}}{\partial y}$ stand for the $z$-component of vorticity and current density, respectively. $W(z), K(z), \Theta(z), Z(z)$ and $X(z)$ are functions of $z$ only.

Using expression (14), equations (9)-(13) in nondimensional form transform to

$$
\begin{gathered}
\left(D^{2}-a^{2}\right)\left[\frac{\sigma}{\varepsilon}+\frac{1}{P_{l}}-\frac{F}{P_{l}}\left(D^{2}-a^{2}\right)\right] W+\frac{g \alpha d^{2}}{v} a^{2} \Theta \\
+\frac{2 \Omega d^{3}}{v \varepsilon} D Z-\frac{\mu_{e} H d}{4 \pi \rho_{0} v}\left(D^{2}-a^{2}\right) D K=0, \\
{\left[\frac{\sigma}{\varepsilon}+\frac{1}{P_{l}}-\frac{F}{P_{l}}\left(D^{2}-a^{2}\right)\right] Z} \\
=\left(\frac{\mu_{e} H d}{4 \pi \rho_{0} v}\right) D X+\left(\frac{2 \Omega d}{v \varepsilon}\right) D W, \\
\left(D^{2}-a^{2}-p_{2} \sigma\right) K=-\left(\frac{H d}{\varepsilon \eta}\right) D W,
\end{gathered}
$$




$$
\begin{gathered}
\left(D^{2}-a^{2}-p_{2} \sigma\right) X=-\left(\frac{H d}{\varepsilon \eta}\right) D Z, \\
\left(D^{2}-a^{2}-E p_{1} \sigma\right) \Theta=-\frac{d^{2}}{k} \frac{g}{c_{p}}(G-1) W,
\end{gathered}
$$

where we have put $a=k d, \sigma=\frac{n d^{2}}{v}$, and $D=\frac{d}{d z} \cdot p_{1}$ $=\frac{v}{\kappa}$ is the Prandtl number, $P_{l}=\frac{k_{1}}{d^{2}}$ is dimensionless medium permeability, $G=\frac{c_{p} \beta}{g}$ is dimension compressibility parameter, $p_{2}=\frac{v}{\eta}$ is the magnetic Prandtl number, and $F=\frac{\mu^{\prime}}{\rho_{0} d^{2} v}$ is the dimensionless couplestress viscosity.

Consider the case where both the boundaries are free as well as perfect conductors of heat, while the adjoining medium is also perfectly conducting. The case of two free boundaries is a little artificial (realistic in stellar atmosphere), but it enables us to find analytical solution and to make some qualitative conclusions. The appropriate boundary conditions, with respect to which equations (15)-(19) must be solved are

$$
W=D^{2} W=0, \quad D Z=0, \quad \Theta=0, \text { at } z=0 \text { and } 1,
$$

$D X=0, \quad K=0$ on a perfectly conducting boundary.

Using the above boundary conditions, it can be shown that all the even order derivatives of $W$ must vanish for $z=0$ and 1 and hence the proper solution of $W$ characterizing the lowest mode is

$$
W=W_{0} \sin \pi z
$$

where $W_{0}$ is a constant.

Eliminating $\Theta, X, Z$ and $K$ between equations (15)-(19) and substituting the proper solution $W=$ $W_{0} \sin \pi z$ in the resultant equation, we obtain the dispersion relation

$$
\begin{array}{r}
R_{1}\left[\frac{G}{G-1}\right]\left(\frac{1+x}{x}\right)\left[\frac{i \sigma_{1}}{\varepsilon}+\frac{1}{P}+\frac{\pi^{2} F}{P}(1+x)\right] \\
\times\left[1+x+i E p_{1} \sigma_{1}\right]+Q_{1}\left(\frac{1+x}{x}\right) \frac{\left(1+x+i E p_{1} \sigma_{1}\right)}{\left(1+x+i p_{2} \sigma_{1}\right)}
\end{array}
$$

$$
+T_{A_{1}}\left[\frac{\left(1+x+i E p_{1} \sigma_{1}\right)\left(1+x+i p_{2} \sigma_{1}\right)}{\left\{\left(\frac{i \sigma_{1}}{\varepsilon}+\frac{1}{P}+\frac{\pi^{2} F}{P}(1+x)\left(1+x+i p_{2} \sigma_{1}\right)+Q_{1}\right)\right\}}\right]
$$

where $R=\frac{g \alpha \beta d^{4}}{v \kappa \pi^{4}}, Q_{1}=\frac{\mu_{e} H^{2} d^{2}}{4 \pi \rho_{0} v \eta \varepsilon \pi^{2}}, T_{A_{1}}=\left(\frac{2 \Omega d^{2}}{\varepsilon v \pi^{2}}\right)^{2}$, $x=\frac{a^{2}}{\pi^{2}}, i \sigma_{1}=\frac{\sigma}{\pi^{2}}$, and $P=\pi^{2} P_{l}$.

Equation (22) includes the effects of magnetic field, rotation, couple-stress and medium permeability on the couple-stress fluid heated from below in a porous medium in the presence of uniform vertical magnetic field and uniform vertical rotation. In the absence of rotation $\left(T_{A_{1}}=0\right)$, the dispersion relation (22) reduces to that by Sharma and Thakur [13].

\section{THE STATIONARY CONVECTION}

When the instability sets in as stationary convection, the marginal state will be characterized by $\sigma=0$. Putting $\sigma=0$, the dispersion relation (22) reduces to

$$
\begin{gathered}
R_{1}\left[\frac{G}{G-1}\right]\left(\frac{1+x}{x}\right)\left[\left\{\frac{1+x}{P}+\frac{\pi^{2} F}{P}(1+x)^{2}\right\}+Q_{1}\right] \\
+T_{A_{1}} \frac{(1+x)^{2}}{x\left[\left\{\frac{1+x}{P}+\frac{\pi^{2} F}{P}(1+x)^{2}\right\}+Q_{1}\right]} .
\end{gathered}
$$

Equation (23) expresses the modified Rayleigh number $R_{1}$ as a function of the dimensionless wave number $x$ and the parameters $Q_{1}, G, P, F$ and $T_{A_{1}}$. For fixed $F, G, T_{A_{1}}, Q_{1}$, let $G$ be also kept constant. Then we find that

$$
\bar{R}_{c}=\left(\frac{G}{G-1}\right) R_{c}
$$

where $\bar{R}_{c}$ and $R_{c}$ denote critical Rayleigh number in the presence and absence of compressibility.

To study the effects of rotation, magnetic field, couple-stress parameter and medium permeability, we 
examine the nature of $\frac{d R_{1}}{d T_{A_{1}}}, \frac{d R_{1}}{d Q_{1}}, \frac{d R_{1}}{d F}$ and $\frac{d R_{1}}{d P}$ analytically.

Equation (23) yields

$$
\begin{aligned}
\frac{d R_{1}}{d T_{A_{1}}} & =\left[\frac{G}{G-1}\right] \frac{(1+x)^{2} P}{x\left\{(1+x)+\pi^{2} F(1+x)^{2}\right\}+P Q_{1}}, \\
& \times\left[1-\frac{\frac{d R_{1}}{d Q_{1}}=\left[\frac{G}{G-1}\right]\left(\frac{1+x}{x}\right)}{\left\{(1+x)+\pi^{2} F(1+x)^{2}+P Q_{1}\right\}^{2}}\right], \\
& \times\left[1-\frac{\frac{d R_{1}}{d F}=\left[\frac{G}{G-1}\right] \frac{\pi^{2}(1+x)^{3}}{x P}}{\left\{(1+x)+\pi^{2} F(1+x)^{2}+P Q_{1}\right\}^{2}}\right], \\
& \frac{d R_{1}}{d F}\left[\frac{G}{G-1}\right] \frac{(1+x)^{2}\left\{1+\pi^{2} F(1+x)\right\}}{x P^{2}} \\
& \times\left[1-\frac{(1+x) P^{2} T_{A_{1}}}{\left\{(1+x)+\pi^{2} F(1+x)^{2}+P Q_{1}\right\}^{2}}\right] .
\end{aligned}
$$

It is clear from equation (25) that, for a stationary convection, the rotation postpones the onset of convection in a couple-stress fluid heated from below in a porous medium in the presence of magnetic field.

It is evident from equation (26) and equation (27) that, for a stationary convection, the magnetic field and the couple-stress postpone the onset of convection in the absence of rotation and also postpone the onset of convection in the presence of rotation if

$$
T_{A_{1}}<\frac{\left\{(1+x)+\pi^{2} F(1+x)^{2}+P Q_{1}\right\}^{2}}{(1+x) P^{2}}
$$

whereas the magnetic field and the couple-stress hasten the onset of convection if

$$
T_{A_{1}}>\frac{\left\{(1+x)+\pi^{2} F(1+x)^{2}+P Q_{1}\right\}^{2}}{(1+x) P^{2}} .
$$

It is evident from equation (28) that, for a stationary convection, the medium permeability hastens the onset of convection in the absence of rotation and also hastens the onset of convection in the presence of rotation if

$$
T_{A_{1}}<\frac{\left\{(1+x)+\pi^{2} F(1+x)^{2}+P Q_{1}\right\}^{2}}{(1+x) P^{2}}
$$

whereas the medium permeability postpones the onset of convection if

$$
T_{A_{1}}>\frac{\left\{(1+x)+\pi^{2} F(1+x)^{2}+P Q_{1}\right\}^{2}}{(1+x) P^{2}},
$$

The dispersion relation (23) is analysed numerically. In Fig. $1, R_{1}$ is plotted against $x$ for $T_{A_{1}}=100$, 200, 300; $P=2, F=2$ and $Q_{1}=2$. It is clear that the rotation postpones the onset of convection in a couple-stress fluid heated from below in a porous medium in the presence of magnetic field as the Rayleigh number increases with increase in rotation parameter. In Fig. 2, $R_{1}$ is plotted against $x$ for $Q_{1}=2$, 4, 6; $P=10, F=2$ and $T_{A_{1}}=0$. Here, we also find that the magnetic field postpones the onset of convection in the absence of rotation for all wave numbers as the Rayleigh number increases with an increase in magnetic field parameter.

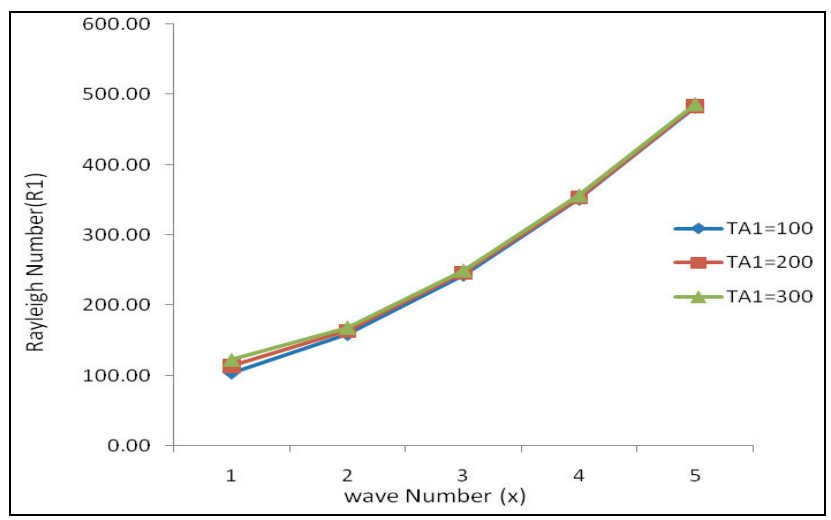

Fig. 1

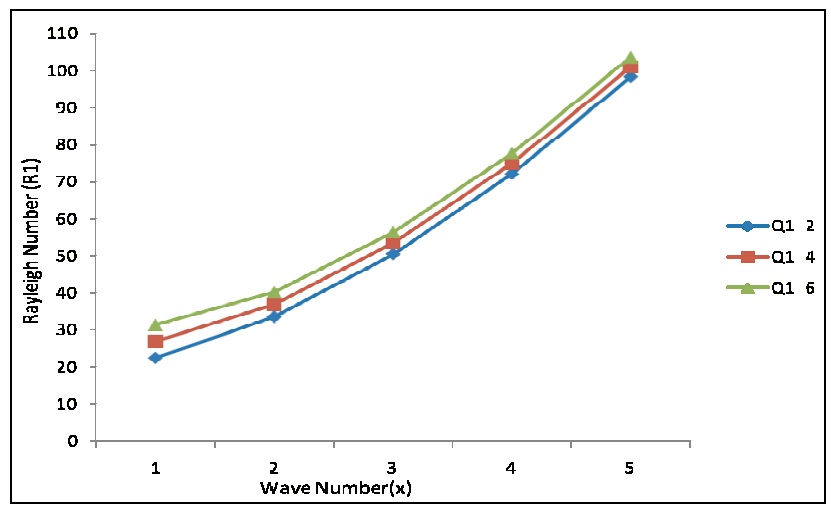

Fig. 2

Also in Fig. 3, $R_{1}$ is plotted against $x$ for $Q_{1}=2,4$, 6,$8 ; P=10, F=2$ and $T_{A_{1}}=100$. Here we find that the magnetic field hastens the onset of convection in the presence of rotation for small wave numbers only as the Rayleigh number decreases with an increase in 
magnetic field parameter and postpones the onset of convection in the absence of rotation for higher wave numbers. In Fig. 4, $R_{1}$ is plotted against $x$ for $F=1,2,3$, $Q_{1}=2, P=20$ and $T_{A_{1}}=0$. Here, we also find that the couple-stress postpones the onset of convection in the absence of rotation for all wave numbers as the Rayleigh number increases with an increase in couplestress parameter.

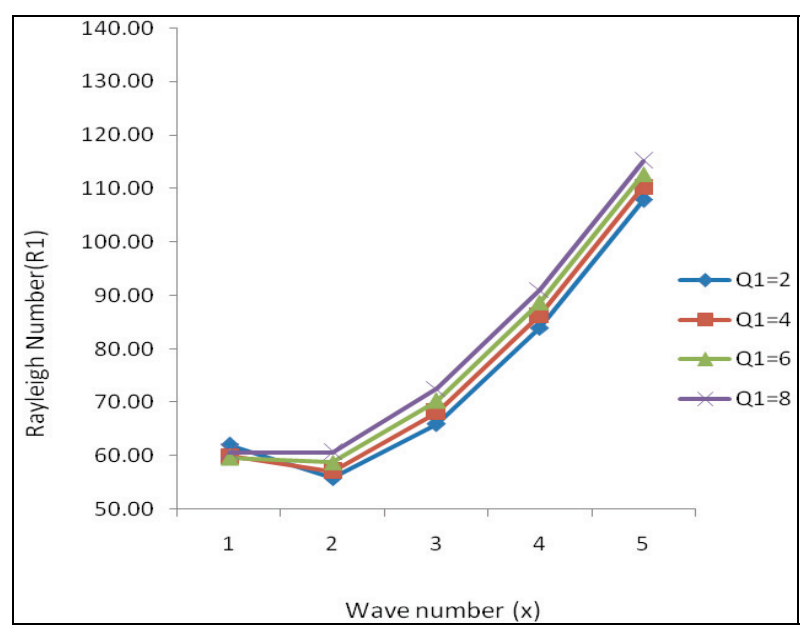

Fig. 3

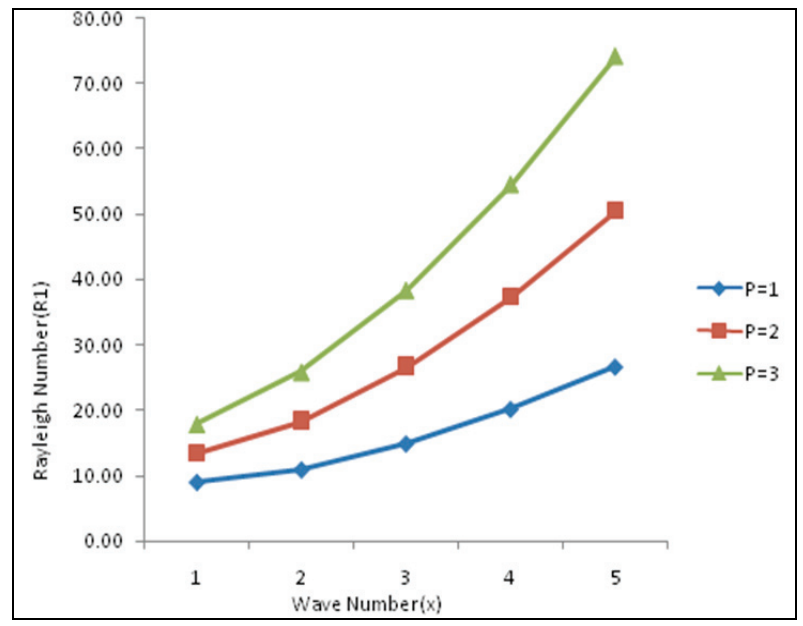

Fig. 4

In Fig. 5, $R_{1}$ is plotted against $x$ for $F=1,2,3$, $Q_{1}=2, P=20$ and $T_{A_{1}}=100$. Here, we also find that the couple-stress hastens the onset of convection in the presence of rotation for small wave numbers as the Rayleigh number decreases with the increase in couple-stress parameter and it postpones the onset of convection in the absence of rotation for higher wave number. In Fig. $6, R_{1}$ is plotted against $x$ for $P=20$, $30,40, Q_{1}=2, F=2$ and $T_{A_{1}}=0$. It is clear that the medium permeability hastens the onset of convection in the presence of rotation for all wave numbers as the Rayleigh number decreases with an increases in medium permeability parameter.

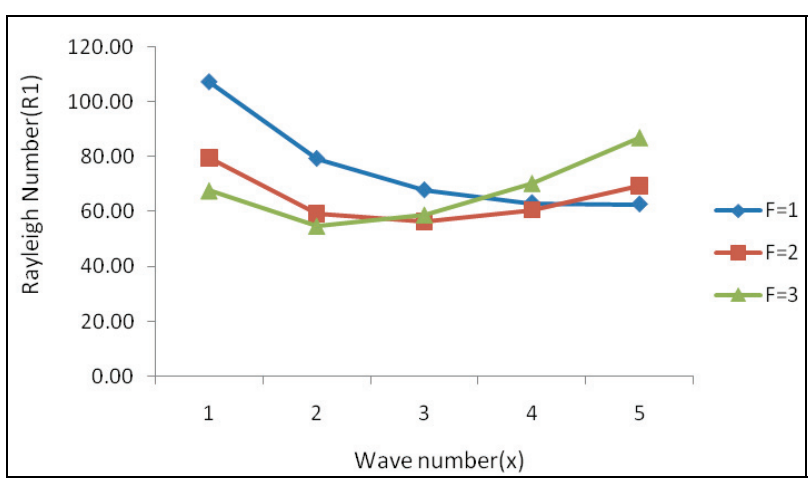

Fig. 5

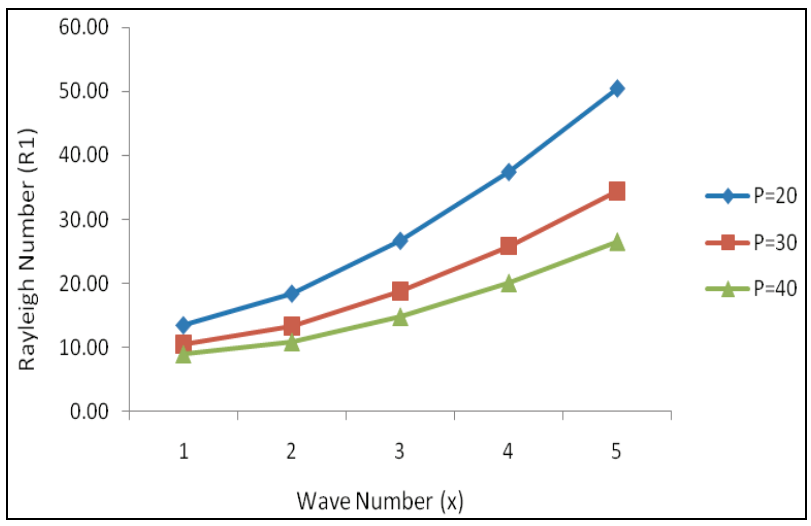

Fig. 6

In Fig. 7, $R_{1}$ is plotted against $x$ for $P=20,30,40$, 50, $Q_{1}=2, F=2$ and $T_{A_{1}}=100$. Here we find that the medium permeability postpones the onset of convection in the presence of rotation for small wave numbers as the Rayleigh number increases with an increase in medium permeability parameter and medium permeability hastens the onset of convection in the presence of rotation for higher wave numbers as the Rayleigh number increases with an increase in medium permeability parameter.

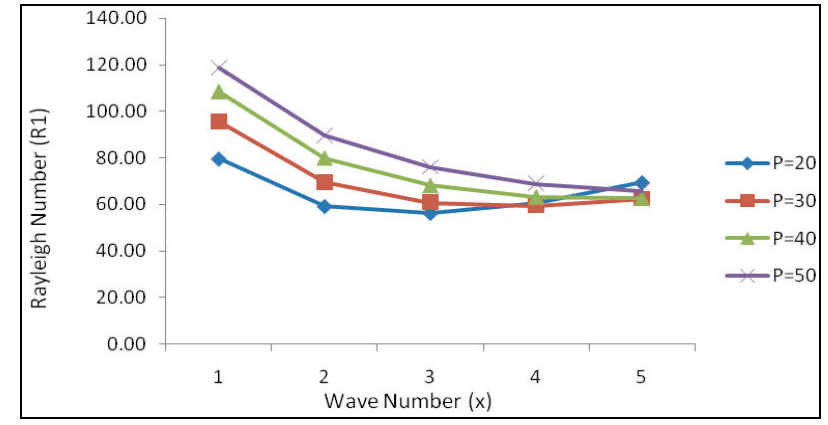

Fig. 7 


\section{STABILITY OF THE SYSTEM AND OSCILLATORY MODES}

Here, we examine the possibility of oscillatory modes, on a stability problem due to the presence of rotation and magnetic field. Multiplying (15) by $W^{*}$, which is the complex conjugate of $W$, and using (16)-(19) together with the boundary conditions (20), we obtain

$$
\begin{gathered}
F I_{1}+\left(1+P_{l} \frac{\sigma}{\varepsilon}\right) I_{2}-\left(\frac{g \alpha \kappa a^{2}}{v \beta}\left[\frac{G}{G-1}\right] P_{l}\right) \\
\times\left[I_{3}+E p_{1} \sigma^{*} I_{4}\right]+\frac{\mu_{e} \varepsilon \eta}{4 \pi \rho_{0} v} P_{l}\left(I_{5}+p_{2} \sigma^{*} I_{6}\right) \\
+\frac{\mu_{e} \varepsilon \eta d^{2}}{4 \pi \rho_{0} v} P_{l}\left(I_{9}+p_{2} \sigma I_{10}\right)+d^{2}\left[\left(1+P_{l} \frac{\sigma^{*}}{\varepsilon}\right) I_{8}+F I_{7}\right]
\end{gathered}
$$

where

$$
\begin{gathered}
I_{1}=\int_{0}^{1}\left(\left|D^{2} W\right|^{2}+2 a^{2}|D W|^{2}+a^{4}|W|^{2}\right) d z, \\
I_{2}=\int_{0}^{1}\left(|D W|^{2}+a^{2}|W|^{2}\right) d z, \\
I_{3}=\int_{0}^{1}\left(|D \Theta|^{2}+a^{2}|\Theta|^{2}\right) d z, \\
I_{5}=\int_{0}^{1}\left(\left|D^{2} K\right|^{2}+2 a^{2}|D K|^{2}+a^{4}|K|^{2}\right) d z, \\
I_{6}=\int_{0}^{1}\left(|D K|^{2}+a^{2}|K|^{2}\right) d z, \\
I_{7}=\int_{0}^{1}\left(|D Z|^{2}+a^{2}|Z|^{2}\right) d z \\
I_{9}=\int_{0}^{1}\left(|D X|^{2}+a^{2}|X|^{2}\right) d z \\
I_{8}=\int_{0}^{1}\left(|Z|^{2}\right) d z
\end{gathered}
$$

$$
I_{10}=\int_{0}^{1}\left(|X|^{2}\right) d z .
$$

The integrals $I_{1}, \ldots, I_{10}$ are all positive-definite. Putting $\sigma=\sigma_{r}+i \sigma_{\mathrm{I}}$ where $\sigma_{r}, \sigma_{\mathrm{I}}$ are real and equating the real and imaginary parts of equation (31), we obtain

$$
\begin{aligned}
\sigma_{r}\left(\frac{I_{2}}{\varepsilon}-\right. & \left.\frac{g \alpha \kappa a^{2}}{v \beta} E p_{1} I_{4}+\frac{\mu_{e} \varepsilon \eta}{4 \pi \rho_{0} v} p_{2}\left(I_{6}+d^{2} I_{10}\right)+\frac{d^{2}}{\varepsilon} I_{8}\right) \\
= & -\left(\frac{F}{P_{l}} I_{1}+\frac{1}{P_{l}} I_{2}-\frac{g \alpha \kappa a^{2}}{v \beta}\left[\frac{G}{G-1}\right] I_{3}\right. \\
+ & \frac{\mu_{e} \varepsilon \eta}{4 \pi \rho_{0} v}\left(I_{5}+d^{2} I_{9}\right)+\frac{d^{2}}{P_{l}}\left(I_{8}+F I_{7}\right), \\
& \quad \frac{\mu_{e} \varepsilon \eta}{4 \pi \rho_{0} v} p_{2}\left(\frac{I_{2}}{\varepsilon}+\frac{g \alpha \kappa a^{2}}{v \beta}\left[\frac{G}{G-1}\right] E p_{1} I_{4}\right.
\end{aligned}
$$

It is evident from equation (33) that $\sigma_{r}$ is either positive or negative. The system is, therefore, either stable or unstable. It is clear from equation (34) that $\sigma_{i}$ may be either zero or non-zero, meaning that the modes may be either non-oscillatory or oscillatory. The oscillatory modes are introduced due to the presence of rotation and the magnetic field, which were non-existent in their absence.

\section{THE CASE OF OVERSTABILITY}

Here, we discuss the possibility of whether instability may occur as overstability. Since we wish to determine the critical Rayleigh number for the onset of instability via a state of pure oscillations, it suffices to find conditions for which (22) will admit of solutions with $\sigma_{1}$ real. Equating real and imaginary parts of equation (22) and eliminating $R_{1}$ between them, we obtain

$$
A_{1} c_{1}^{3}+A_{2} c_{1}^{2}+A_{1} c_{1}+A_{0}=0
$$

where we have put $c_{1}=\sigma_{1}^{2}, b=1+x$ and 


$$
\begin{gathered}
A_{0}=\left[\frac{\pi^{4} F^{2}}{P^{2}}\left\{\frac{1}{\varepsilon}+\frac{E p_{1} \pi^{2} F}{P}\right\} b^{8}+\frac{\pi^{2} F}{P^{2}}\left\{\frac{2}{\varepsilon}+\frac{3 E p_{1} \pi^{2} F}{P}\right\} b^{7}\right. \\
+\left\{\frac{\pi^{4} F^{2} Q_{1}}{P^{2}}\left(3 E p_{1}-p_{2}\right)+\frac{1}{P^{2}}\left\{\frac{1}{\varepsilon}+\frac{3 E p_{1} \pi^{2} F}{P}\right\}+\frac{2 Q_{1} \pi^{2} F}{\varepsilon P}\right\} b^{6} \\
+\left\{\frac{2 \pi^{2} F Q_{1}}{P^{2}}\left(3 E p_{1}-p_{2}\right)+\left\{\frac{E p_{1}}{P^{3}}-\frac{T_{A_{1}}}{\varepsilon}\right\}+\frac{1}{P}\left\{\frac{2 Q_{1}}{\varepsilon}+E p_{1} \pi^{2} F T_{A_{1}}\right\}\right\} b^{5} \\
+\left\{\frac{\pi^{2} F Q_{1}^{2}}{P^{2}}\left(3 E p_{1}-2 p_{2}\right)+\frac{Q_{1}}{P^{2}}\left(3 E p_{1}-p_{2}\right)+\frac{Q_{1}^{2}}{\varepsilon}+\frac{E p_{1} T_{A_{1}}}{P}\right\} b^{4} \\
\left.+\left\{\frac{Q_{1}^{2}}{P}\left(3 E p_{1}-2 p_{2}\right)+Q_{1} T_{A_{1}}\left(E p_{1}+p_{2}\right)\right\} b^{3}+\left\{Q_{1}^{2}\left(E p_{1}-p_{2}\right)\right\} b^{2}\right]
\end{gathered}
$$

$$
A_{3}=\frac{p_{2}^{4}}{\varepsilon^{2}}\left[\left\{\frac{1}{\varepsilon}+\frac{E p_{1} \pi^{2} F}{P}\right\} b^{2}+\left\{\frac{E p_{1}}{P}\right\} b\right]
$$

Since $\sigma_{1}$ is real for overstability, the values $c_{1}=\sigma_{1}^{2}$ of equation (35) are positive, so the product of the roots of equation (35) is positive but this is impossible if $A_{0}>0$ (since product of the roots of equation (35) is $-\frac{A_{0}}{A_{3}}$ and $A_{3}>0$ ). $A_{0}>0$ is therefore a sufficient condition for the non-existence of over stability.

From equation (36) it is clear that $A_{0}$ is always positive if

$$
E p_{1}>p_{2} \quad \text { and } \quad T_{A_{1}}<\frac{E p_{1} \varepsilon}{P^{3}}
$$

i.e.,

$$
E \eta>\kappa \text { and } \kappa<E\left(\frac{v \varepsilon}{k_{1}}\right)^{3}\left(\frac{d}{2 \Omega \pi}\right)^{2}
$$

which imply that

$$
\kappa<\min \left[E \eta, E\left(\frac{v \varepsilon}{k_{1}}\right)^{3}\left(\frac{d}{2 \Omega \pi}\right)^{2}\right] .
$$

The condition is therefore a sufficient condition for the non-existence of overstability, the violation of which does not necessarily imply the occurrence of overstability.

\section{CONCLUSION}

The effects of magnetic field, rotation, medium permeability and couple stress parameter on couple stress fluid heated from below have been investigated. The main conclusions are:

(I) For a stationary convection, the magnetic field and the couple-stress postpone the onset of convection in the absence of rotation and also postpone the onset of convection in the presence of rotation. Whereas, for a stationary convection, the medium permeability hastens as well as postpones the onset of convection in the absence of rotation and also in the presence of rotation.

(II) The oscillatory modes are introduced due to the presence of rotation and the magnetic field, which were non existent in their absence.

(III) It is clear that the effect of compressibility is to postpone the onset of instability.

(IV) A sufficient condition for the non-existence of overstability, the violation of which does not necessarily imply the occurrence of overstability.

\section{REFERENCES}

[1] BANYAL A.S., A mathematical theorem on the onset of stationary convection in the couple-stress fluid, Journal of Applied Fluid Mechanics, 2013, Vol. 6, No. 2.

[2] BRAKKE M.K., Zone electrophoresis of dyes proteins viruses in density gradient columns of sucrose solutions, Arch. Biochem. Biophys., 1955, 55, 175.

[3] ChandraseKhar S., Hydrodynamic and Hydromagnetic Stability, Dover Publication, New York, 1981. 
[4] Joseph D.D., Stability of Fluid Motions, Vol. II, Springer Verlag, Berlin 1976.

[5] KUMAR P., Thermosolutal magneto-rotatory convection in couple stress fluid through porous medium, Journal of Applied Fluid Mechanics, 2012, Vol. 5, No. 4.

[6] LinDEN P.F., Salt and fingers in a study shear flow, Geophy. Fluid Dynamics, 1974, 6, 1.

[7] Nason P., Schumaker V., Halsall B., Schwedes J., Formation of a streaming convective disturbance which may occur at one gravity during preparation of samples for zone centrifugation, Biopolymers, 1969, 7, 241.

[8] RANA C. GIAN, The onset of thermal convection in couple-stress fluid in hydromagnetics saturating a porous medium, Bulletin of the Polish Academy of Sciences, 2014, Vol. 62, No. 2.

[9] RUdRAiAh N., CHANDRASHEKRA G., Effect of couple stress on the growth rate of Rayleigh Taylor instability at the interface in a finite thickness couple stress fluids, Journal of Applied Fluid Mechanics, 2010, Vol. 3, No. 1.

[10] Sharma R.C., Thermal instability in compressible fluids in the presence of rotation and magnetic field, J. Math. Anal. Appl., 1977, 60, 227.

[11] Sharma R.C., Mehta C.B., Thermosolutal convection in compressible, rotating, couple stress fluid, Indian J. Phys., 2005, 79(2), 161.
[12] Sharma R.C., Sharma S., On couple-stress fluid heated from below in porous medium, Indian J. Phys., 2001, 75(2), 137.

[13] Sharma R.C., ThakuR K.D., On couple-stress fluid heated from below in porous medium in hydromagnetics, Czech. J. Phys., 2000, 50, 753.

[14] Singh M., Menta C.B., Thermosolutal convection incompressible, rotating couple stress fluid in the presence of magnetic fluid, AASRFC, 2012, 3(6), 3459.

[15] Singh M., Menta C.B., On compressible couple fluid heated and sotuted from below in porous medium in presence of rotation and magnetic field, JMET, 2013, 1(1), 21.

[16] Spiegel E.A., Veronis G., On the Boussinesq approximation for a compressible fluid, The Astrophys. J., 1960, 131, 442.

[17] Stokes V.K., Couple-stresses in fluids, Phys. Fluids, 1966, 9, 1709.

[18] Sunil, Sharma R.C., PAL M., On a couple stress fluid heated from below in a porous medium in the presence of a magnetic field and rotation, Journal of Porous Media, USA, 2002, 149.

[19] WALICKI E., WaLICKA A., Inertia effect in the squeeze film of a couple-stress fluid in biological bearings, Appl. Engng., 1999, 4. 